\title{
Tubercular Pyomyositis: An Uncommon Presentation of Extrapulmonary Tuberculosis
}

\author{
Satish Swain ${ }^{1}$, Akansha Didwania², Manish Soneja ${ }^{3}$
}

\begin{abstract}
Background:Tubercular pyomyositis is a rare entity in immunocompetent induvial, and involvement of head and neck region is very uncommon. It usually presents as diffuse swelling with some tenderness and other constitutional symptoms of tuberculosis (like a low-grade fever, loss of appetite, and weight loss). It can also mimic other entities like a pyogenic abscess, malignancy (sarcoma), benign soft tissue tumors, cervical lymphadenitis, and hematoma; which can pose a diagnostic challenge.

Case description: We here present a case of a 22-year-old male who came with complaints of neck swelling with low-grade fever and weight loss for about a month duration. A detailed clinical history and examination helped us to narrow down the differential. Finally radiological imaging and microbiological test aided in clenching the diagnosis and helping with further management. The patient was diagnosed with tubercular pyomyositis of cervical muscles and was successfully treated with first-line anti-tubercular therapy.

Keywords: Extrapulmonary tuberculosis, Infectious disease, Musculoskeletal infection.

The Journal of Medical Sciences (2021): 10.5005/jp-journals-10045-00164
\end{abstract}

\section{BACKGROUND}

Tuberculosis is now a global concern and a serious threat to public health around the globe, especially the underdeveloped and developing nations. Tubercular pyomyositis is an uncommon form of extrapulmonary tuberculosis involving skeletal muscles. It has varied clinical presentations which sometimes can pose a diagnostic challenge in clinical practice.

\section{Case Description}

A 22-year-old male, non-smoker and with no prior comorbidities presented with a swelling over the left side of the neck which was insidious in onset and gradually progressing in size over the last 1.5 months. The patient also has a history of intermittent low grade fever (99.9-100 ${ }^{\circ}$ Fahrenheit) with weight loss of over $7 \mathrm{~kg}$ over past 2-3 months. On local examination, there was a mildly tender, nonpulsatile, swelling roughly measuring $4 \mathrm{~cm}$ by $5 \mathrm{~cm}$ (Fig. 1A) over the left posterior triangle of the neck with mild erythema over the overlying skin. There was no palpable lymphadenopathy noted, while the examination of the respiratory, cardiovascular, abdominal, and nervous systems was unremarkable. Routine hematological and biochemical parameters were within normal limits, apart from an elevated erythrocytic sedimentation rate (ESR) of $55 \mathrm{~mm} /$ hour. The chest radiograph was also normal. The patient also tested negative for human immunodeficiency virus (HIV), hepatitis B (HBsAg), and hepatitis C (Anti-HCV). An initial ultrasonography (USG) examination was done which revealed a well-defined predominately hypoechoic lesion. Later, a contrast-enhanced computed tomography (CECT) scan of the neck was done to further characterize the swelling which showed an enhancing collection in left paravertebral muscles of the neck without any boney involvement (Figs $1 \mathrm{~B}$ and C). The collection was aspirated under ultrasound guidance and was sent for bacterial, tubercular, and fungal stains and cultures. The bacterial and fungal workup (Gram stain, $\mathrm{KOH}$ stain) did not yield anything but ZiehlNeelsen (ZN) stain showed acid-fast bacilli. Gene Xpert ${ }^{\mathrm{TM}}$ of the same also detected Mycobacterium tuberculosis with rifampicin sensitivity.
${ }^{1}$ Department of Anaesthesiology, All India Institute of Medical Science, New Delhi, India

2,3 Department of Medicine, All India Institute of Medical Science, New Delhi, India

Corresponding Author: Manish Soneja, Department of Medicine, All India Institute of Medical Science, New Delhi, India, Phone: +91 7678374352, e-mail: manishsoneja@gmail.com

How to cite this article: Swain S, Didwania A, Soneja M. Tubercular Pyomyositis: An Uncommon Presentation of Extrapulmonary Tuberculosis. J Med Sci 2021;7(1):11-13.

Source of support: Nil

Conflict of interest: None

The patient was started on weight-based first-line anti-tubercular therapy [Isoniazid (H), Rifampicin (R), Pyrazinamide (Z), Ethambutol (E)] with a final diagnosis of cervical paravertebral pyomyositis of tubercular etiology. The patient is currently following up with us in the infectious disease clinic of our institute and is doing well.

\section{Discussion}

Pyomyositis is a purulent infection of skeletal muscle, usually with abscess formation and most commonly caused by Staphylococcus ( $90 \%$ cases), but tubercular pyomyositis is a very uncommon entity. ${ }^{1}$ In a study by Wang et al., out of 1,153 culture-positive tuberculosis, $1.8 \%$ had tuberculous myositis and none of them had involvement of head and neck muscles. ${ }^{2}$ Other cases of tubercular pyomyositis are limited to case reports and mostly in a backdrop of an immunocompromised condition (immunosuppressive therapy, corticosteroids, retroviral disease, or renal failure). ${ }^{3,4}$ Skeletal muscles are usually resistant to infection from tubercular bacilli, although the exact mechanisms are not known, it has been implicated to high lactic acid concentration, poor oxygen content, and absence of reticuloendothelial cells and lymphatic tissue in 

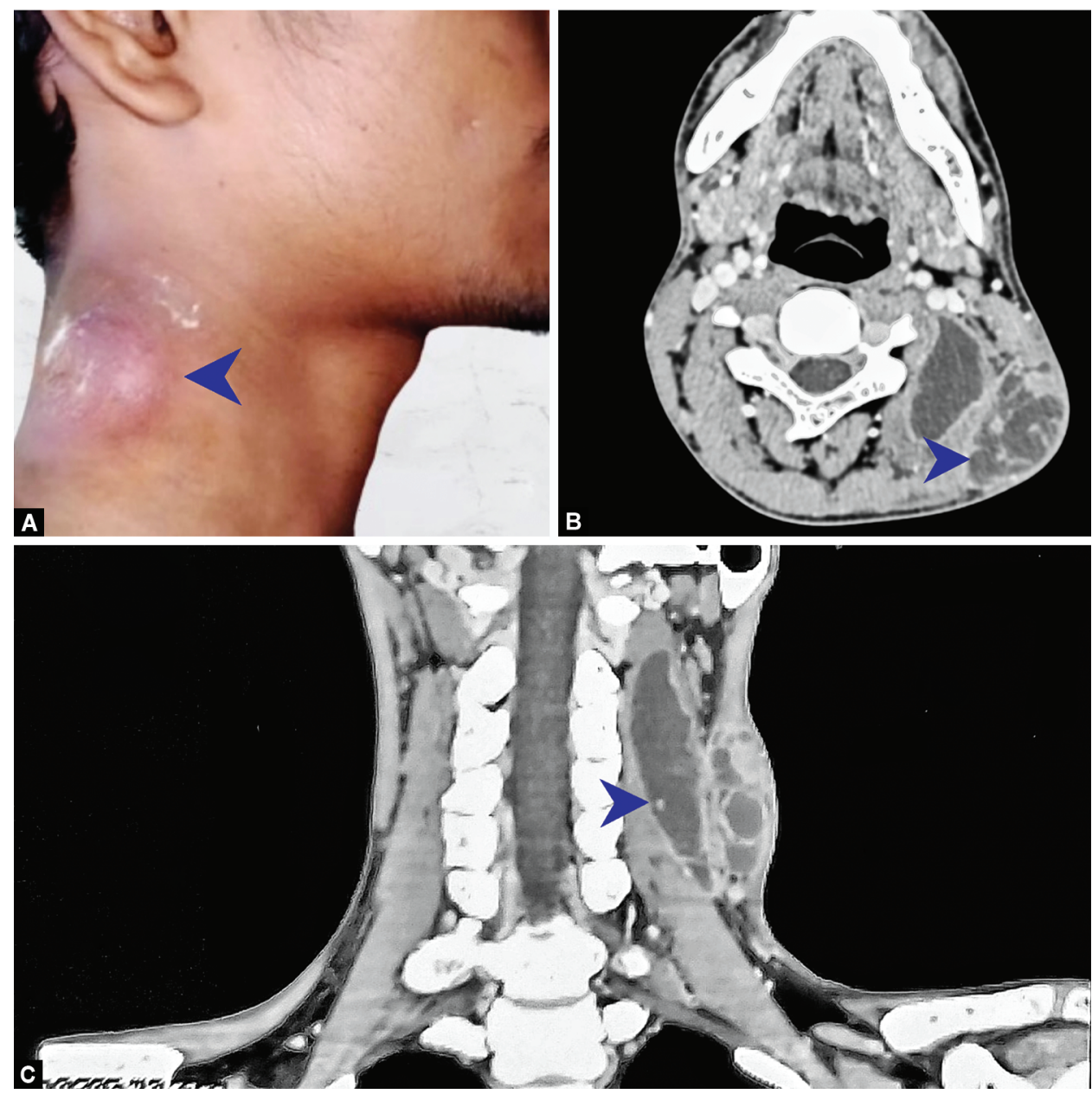

Figs 1 A to C: (A) Image of the swelling on the left posterior triangle of neck (blue arrowhead); (B) Computed tomography image of the neck (sagittal view) extension of a collection into left posterior cervical space (blue arrowhead); (C) Computed tomography image of the neck (coronal view) showing peripherally enhancing collection seen in left paravertebral muscles and left trapezius (blue arrowhead)

muscles. ${ }^{5}$ Mycobacterium reaches skeletal muscles via extension from adjacent structures (like bone, synovial membrane, or tendon sheaths), direct inoculation (needles, trauma), or via lymphatic or hematogenous seeding from a distant site. The clinical picture in bacterial pyomyositis is divided into three stages: invasive (variable fever, painful muscle swelling, and minimal systemic symptoms), suppurative (high spiky fever, with more severe systemic symptoms and classical signs of abscess), and late (bacteremia, septicemia, septic shock, and metastatic complications). ${ }^{6}$ But these various clinical stages of and classical signs (like high-grade fever, muscle tenderness, and leukocytosis) pyogenic pyomyositis may not be apparent in tubercular etiology, which may pose a diagnostic challenge. ${ }^{4}$ This entity may mimic other clinical conditions like a pyogenic abscess, malignancy (sarcoma), benign soft tissue tumors, cervical lymphadenitis, and hematoma; so it becomes important to distinguish between them. Imaging studies (like USG, CT scan, and MRI) are important in making a diagnosis along with microbiological evaluations like stains, culture, and molecular methods (Gene Xpert ${ }^{\mathrm{T}}$, PCR, etc.). ${ }^{5}$ Management comprise anti-tubercular therapy and surgery. Response to standard antitubercular therapy (category I ATT) HRZE for 2 months (induction phase) followed by HRE for 4 months (continuation phase) has a good response. Drug resistance tubercular pyomyositis as detected by Gene Xpert ${ }^{\mathrm{TM}}$, or line probe assay or culture will require longer therapy with second line anti-tubercular agents. ${ }^{7}$ Surgical drainage or aspiration is needed for management as well as for etiological diagnosis (cytopathological or microbiological).

\section{Clinical Significance and Conclusion}

Tubercular pyomyositis is a rare entity and a form of extrapulmonary tuberculosis. This may mimic other clinical conditions which may pose a diagnostic challenge. Radiological and microbiological diagnostic aid as described is very helpful in reaching the diagnosis. Finally, this disease entity has an excellent response to antitubercular therapy.

\section{Patient Consent Statement}

Consent has been taken directly from the patient.

\section{References}

1. Stevens DL, Bisno AL, Chambers HF, et al. Practice guidelines for the diagnosis and management of skin and soft tissue infections: 2014 update by the Infectious Diseases Society of America. Clin Infect Dis 2014;59(2):e10-e52. DOI: 10.1093/cid/ciu444. 
2. Wang JY, Lee LN, Hsueh PR, et al. Tuberculous myositis: a rare but existing clinical entity. Rheumatology 2003;42(7):836-840. DOI: 10.1093/rheumatology/keg228R.

3. Primary Tuberculous Pyomyositis of the Calf Muscles - The American Journal of the Medical Sciences, https://www.amjmedsci. com/article/S0002-9629(16)30281-6/abstract (accessed 3 July 2021).

4. Krishnasamy V, Joseph M. Tuberculous pyomyositis: a rare but serious diagnosis. Case Rep Med 2013;2013:e126952.
5. Simopoulou T, Varna A, Dailiana Z, et al. Tuberculous pyomyositis: a re-emerging entity of many faces. Clin Rheumatol 2016;35(4):11051110. DOI: 10.1007/s10067-014-2564-8.

6. Chauhan S, Jain S, Varma S, et al. Tropical pyomyositis (myositis tropicans): current perspective. Postgrad Med J 2004;80(943):267270. DOI: 10.1136/pgmj.2003.009274.

7. Sharma SK, Ryan H, Khaparde S, et al. Index-TB guidelines: guidelines on extrapulmonary tuberculosis for India. Indian J Med Res 2017;145(4):448-463. DOI: 10.4103/ijmr.IJMR_1950_16. 